\title{
LOW-DIMENSIONAL FILIFORM LIE SUPERALGEBRAS
}

\author{
Marc GILG
}

\begin{abstract}
The aim of this paper is to give a classification up to isomorphism of low dimension filiform Lie superalgebras.
\end{abstract}

\section{Introduction}

There exists a lot of work concerning Lie superalgebras. But less of them are interested in nilpotent Lie superalgebras, although, the case of nilpotent Lie algebras has been well studied, see for example [1]. This fact is a consequence of the development of deformation theory. In this paper, we will focus a particular class of nilpotent Lie superalgebras. We recall some definitions from [3]. We point out the definition of filiform Lie superalgebras. This definition is analogue to the definition of filiform Lie algebras given by Vranceanu [10] and Vergne [9]. The classification of this super-algebras is still a problem, as the classification of filiform Lie algebras over an algebraically closed field of characteristic zero is only done up to dimension 11 [4]. In this paper, we will give a first classification for filiform Lie superalgebras in low dimensions.

\section{Filiform Lie superalgebras}

A $\mathbb{Z}_{2}$-graded vector space $\mathcal{G}=\mathcal{G}_{0} \oplus \mathcal{G}_{1}$ over an algebraically closed field is a Lie superalgebra if there exists a bilinear product [,] over $\mathcal{G}$ such that

$$
\begin{aligned}
& {\left[G_{\alpha}, \mathcal{G}_{\beta}\right] \subset \mathcal{G}_{\alpha+\beta} \bmod 2,} \\
& {\left[g_{\alpha}, g_{\beta}\right]=-(-1)^{\alpha \cdot \beta}\left[g_{\beta}, g_{\alpha}\right]}
\end{aligned}
$$

2000 Mathematics Subject Classification: 17B30, 17B70.

Servicio de Publicaciones. Universidad Complutense. Madrid, 2001 
for all $g_{\alpha} \in \mathcal{G}_{\alpha}$ and $g_{\beta} \in \mathcal{G}_{\beta}$ and satisfying Jacobi's super-relation:

$$
(-1)^{\gamma \cdot \alpha}[A,[B, C]]+(-1)^{\alpha \cdot \beta}[B,[C, A]]+(-1)^{\beta \cdot \gamma}[C,[A, B]]=0
$$

for all $A \in \mathcal{G}_{\alpha}, B \in \mathcal{G}_{\beta}$ and $C \in \mathcal{G}_{\gamma}$.

For such a Lie superalgebra we define the lower central series by

$$
\left\{\begin{array}{l}
C^{0}(\mathcal{G})=\mathcal{G}, \\
C^{i+1}(\mathcal{G})=\left[\mathcal{G}, C^{i}(\mathcal{G})\right]
\end{array}\right.
$$

A Lie superalgebra $\mathcal{G}$ is nilpotent if there exists an integer $n$ such that $C^{n}(\mathcal{G})=\{0\}$.

This definition is not easy to use. Therefore we define the following sequences for a Lie superalgebra $\mathcal{G}=\mathcal{G}_{0} \oplus \mathcal{G}_{1}$ :

$$
C^{0}\left(\mathcal{G}_{0}\right)=\mathcal{G}_{0}, \quad C^{i+1}\left(\mathcal{G}_{0}\right)=\left[\mathcal{G}_{0}, C^{i}\left(\mathcal{G}_{0}\right)\right]
$$

and

$$
C^{0}\left(\mathcal{G}_{1}\right)=\mathcal{G}_{1}, \quad C^{i+1}\left(\mathcal{G}_{1}\right)=\left[\mathcal{G}_{0}, C^{i}\left(\mathcal{G}_{1}\right)\right]
$$

Theorem 2.1. Let $\mathcal{G}=\mathcal{G}_{0} \oplus \mathcal{G}_{1}$ be a Lie superalgebras. Then $\mathcal{G}$ is nilpotent if and only if there exist $(p, q)$ such that $C^{p}\left(\mathcal{G}_{0}\right)=\{0\}$ and $C^{q}\left(\mathcal{G}_{1}\right)=\{0\}$.

Proof. If the Lie superalgebra $\mathcal{G}=\mathcal{G}_{0} \oplus \mathcal{G}_{1}$ is nilpotent it is easy to prove that there exist $(p, q)$ such that $C^{p}\left(\mathcal{G}_{0}\right)=\{0\}$ and $C^{q}\left(\mathcal{G}_{1}\right)=\{0\}$.

For the converse, we use the classical Engel's theorem. Let $\mathcal{G}=$ $\mathcal{G}_{0} \oplus \mathcal{G}_{1}$ be a Lie superalgebra. Assume that there exist $(p, q)$ such that $C^{p}\left(\mathcal{G}_{0}\right)=\{0\}$ and $C^{q}\left(\mathcal{G}_{1}\right)=\{0\}$, then $\operatorname{ad}(X)$ with $X \in \mathcal{G}_{0}$ is nilpotent. We have for $Y \in \mathcal{G}_{1}$ :

$$
\operatorname{ad}(Y) \circ \operatorname{ad}(Y)=\frac{1}{2} a d([Y, Y])
$$

As $[Y, Y]$ is an element of $\mathcal{G}_{0}$, then $\operatorname{ad}([Y, Y])$ is nilpotent. This implies that $\operatorname{ad}(Y)$ is nilpotent for every $Y \in \mathcal{G}_{1}$. Hence $\operatorname{ad}(X)$ and $\operatorname{ad}(Y)$ is nilpotent, using Engel's theorem given in [6], $\mathcal{G}=\mathcal{G}_{0} \oplus \mathcal{G}_{1}$ is a nilpotent Lie superalgebra.

Definition 2.1. Let $\mathcal{G}$ be a nilpotent Lie superalgebra, the super-nilindex of $\mathcal{G}$ is the pair $(p, q)$ such that: $C^{p}\left(\mathcal{G}_{0}\right)=\{0\}, C^{p-1}\left(\mathcal{G}_{0}\right) \neq\{0\}$ and $C^{q}\left(\mathcal{G}_{1}\right)=\{0\}, C^{q-1}\left(\mathcal{G}_{1}\right) \neq\{0\}$. It is and invariant up to isomorphism. 
Definition 2.2 (Filiform Lie superalgebras). Let $\mathcal{G}=\mathcal{G}_{0} \oplus \mathcal{G}_{1}$ be a nilpotent Lie superalgebra with $\operatorname{dim} \mathcal{G}_{0}=n+1$ and $\operatorname{dim} \mathcal{G}_{1}=m$. $\mathcal{G}$ is called filiform if it's super-nilindex is $(n, m)$. We will note $\mathcal{F}_{n, m}$ the set of filiform Lie superalgebras.

Let's define the set $\mathcal{N}_{n, m}^{p, q}$ of Lie superalgebras with $\operatorname{dim} \mathcal{G}_{0}=n+1$, $\operatorname{dim} \mathcal{G}_{1}=m$ and with super-nilindex $\left(k_{0}, k_{1}\right)$ such that $k_{0} \leq p$ and $k_{1} \leq q$. It is obvious that this set can be define by polynomial relations given by the Jacobi relations and the nilpotency relations. This prove that it is a Zariski-closed set of the algebraic variety of nilpotent Lie superalgebras denoted by $\mathcal{N}_{n, m}$.

The set $\mathcal{F}_{n, m}$ of filiform Lie superalgebras can be written as the complementary of a close set:

$$
\mathcal{F}_{n, m}=\mathcal{N}_{n, m} \backslash\left(\mathcal{N}_{n, m}^{n-1, m} \cup \mathcal{N}_{n, m}^{n, m-1}\right)
$$

Hence the set of filiform Lie superalgebras is an open set of the variety of nilpotent Lie superalgebras (see [5]).

\section{Classifications of filiforms over $\mathbb{C}$ in low dimensions}

\subsection{Adapted basis}

Like for the filiform Lie algebras [9], there exists an adapted bases of a filiform Lie superalgebra:

Proposition 3.1. Let $\mathcal{G}=\mathcal{G}_{0} \oplus \mathcal{G}_{1}$ be a filiform Lie superalgebra with $\operatorname{dim} \mathcal{G}_{0}=n+1$ and $\operatorname{dim} \mathcal{G}_{1}=m$. Then there exists a bases $\left\{X_{0}, X_{1}, \ldots X_{n}, Y_{1}, Y_{2}, \ldots Y_{m}\right\}$ of $\mathcal{G}$ with $X_{i} \in \mathcal{G}_{0}$ and $Y_{i} \in \mathcal{G}_{1}$ such that:

$$
\left\{\begin{array}{l}
{\left[X_{0}, X_{i}\right]=X_{i+1} \quad 1 \leq i \leq n-1, \quad\left[X_{0}, X_{n}\right]=0 ;} \\
{\left[X_{1}, X_{2}\right] \in \mathbb{C} . X_{4}+\mathbb{C} . X_{5}+\cdots+\mathbb{C} . X_{n} ;} \\
{\left[X_{0}, Y_{i}\right]=Y_{i+1} \quad 1 \leq i \leq m-1, \quad\left[X_{0}, Y_{m}\right]=0 .}
\end{array}\right.
$$

Such basis are called adapted.

Proof. Let $g r \mathcal{G} \in \mathcal{F}_{n, m}$ be a graded filiform lie superalgebra. The lower central series implies the following graduations:

$$
\begin{aligned}
& g r \mathcal{G}_{0}=W_{1}^{0} \oplus W_{2}^{0} \oplus \cdots \oplus W_{n-1}^{0} \\
& g r \mathcal{G}_{1}=W_{1}^{1} \oplus W_{2}^{1} \oplus \cdots \oplus W_{m-1}^{1}
\end{aligned}
$$


with $\operatorname{dim} W_{1}^{0}=2, \operatorname{dim} W_{i}^{0}=1$ for $2 \leq i \leq n-1$ and $\operatorname{dim} W_{j}^{1}=1$ for $1 \leq j \leq m-1$. Then we have $\left[W_{1}^{0}, W_{i}^{0}\right]=W_{i+1}^{0}$ and $\left[W_{1}^{0}, W_{j}^{1}\right]=W_{j+1}^{1}$.

Let $x_{i} \in W_{i}^{0}$ and $y_{j} \in W_{j}^{1}$ be non zero elements, then if $w \in W_{1}^{0}$,

$$
\begin{aligned}
& {\left[w, x_{i}\right]=\lambda_{i}^{0}(w) x_{i+1}} \\
& {\left[w, y_{j}\right]=\lambda_{j}^{1}(w) y_{j+1}}
\end{aligned}
$$

the maps $w \rightarrow \lambda_{i}^{0}(w)$ and $w \rightarrow \lambda_{j}^{1}(w)$ are non zero linear forms on $W_{1}^{0}$, because $\left[W_{1}^{0}, W_{i}^{0}\right]=W_{i+1}^{0}$ and $\left[W_{1}^{0}, W_{j}^{1}\right]=W_{j+1}^{1}$.

As the field is $\mathbb{C}$, there exists $x_{0}$ in $W_{1}^{0}$ such that $\lambda_{i}^{0}(w) \neq 0$ for $1 \leq$ $i \leq n-1$ and $\lambda_{j}^{1}(w) \neq 0$ for $1 \leq j \leq m-1$. Hence it exist a bases $X_{0}, X_{1}, \ldots, X_{n}$ and $Y_{1}, Y_{2}, \ldots, Y_{m}$ with $X_{i}$ ( resp. $Y_{j}$ ) multiple of $x_{i}$ (resp. $y_{j}$ ) such that:

$$
\begin{aligned}
{\left[X_{0}, X_{i}\right] } & =X_{i+1} \text { for } 1 \leq i \leq n-1 \\
{\left[X_{0}, Y_{j}\right] } & =Y_{j+1} \text { for } 1 \leq j \leq m-1 \\
{\left[X_{1}, X_{2}\right] } & =a X_{3} \text { for } a \in \mathbb{C}
\end{aligned}
$$

Substitute $X_{1}$ by $X_{1}-a X_{0}$ we get the adapted bases of $g r \mathcal{G}$ :

$$
\begin{aligned}
{\left[X_{0}, X_{i}\right] } & =X_{i+1} \text { for } 1 \leq i \leq n-1 \\
{\left[X_{0}, Y_{j}\right] } & =Y_{j+1} \text { for } 1 \leq j \leq m-1 \\
{\left[X_{1}, X_{2}\right] } & =0
\end{aligned}
$$

If the Lie superalgebras is not graded, then we introduce a graded Lie superalgebra, as it was done for Lie algebras in [9].

\subsection{Adapted changes of basis}

Definition 3.1. Let $f$ be a graded change of bases of a filiform Lie superalgebra $\mathcal{G} . f$ is an adapted changes of bases if $f$ is Lie superalgebra homomorphism and if the image of an adapted bases of $\mathcal{G}$ is an adapted bases.

Proposition 3.2. Let $\mathcal{G}=\mathcal{G}_{0} \oplus \mathcal{G}_{1}$ be a filiform Lie superalgebra. Let $f=f_{0}+f_{1}$ be an adapted change of basis of $\mathcal{G}$. Then $f_{0}$ is an adapted change of bases of the filiform Lie algebra $\mathcal{G}_{0}$ and $f_{1}$ satisfies:

$$
\left\{\begin{array}{l}
f_{1}\left(Y_{1}\right)=d_{1} Y_{1}+d_{2} Y_{2}+\cdots+d_{m} Y_{m} \\
f_{1}\left(Y_{i}\right)=\left[f_{0}\left(X_{0}\right), f_{1}\left(Y_{i-1}\right)\right] \quad 2 \leq i \leq m
\end{array}\right.
$$


with the condition:

$$
d_{1} \prod_{j=1}^{m-1} A_{j} \neq 0
$$

where the $A_{j}$ 's are defined by:

$$
A_{j}=a_{0}+\sum_{k=0}^{j-1}(-1)^{k} C_{j-1}^{k}\left(\sum_{i=1}^{n-k} a_{i} \cdot r_{i+k, 2+k}\right)
$$

and the product of $\mathcal{G}$ is given in the adapted bases by

$$
\left[X_{i}, Y_{1}\right]=\sum_{s=2}^{m} r_{i, s} Y_{s} \quad 1 \leq i \leq n
$$

Proof. The proof can be found in [3]. We give a sketch of it.

First, we establish that $f_{0}$ is an adapted change of bases for Lie algebras as in [4]. The we set:

$$
\left\{\begin{array}{l}
f_{0}\left(X_{0}\right)=a_{0} X_{0}+a_{1} X_{1}+\ldots a_{n} X_{n} \\
f_{1}\left(Y_{1}\right)=d_{1} Y_{1}+d_{2} Y_{2}+\ldots d_{m} Y_{m}
\end{array}\right.
$$

and assuming $f\left(Y_{t}\right)=\left[f\left(X_{0}\right), f\left(Y_{t-1}\right)\right]$ for $2 \leq t \leq m$ we prove by induction on $t$ that:

$$
f\left(Y_{t}\right)=d_{1} \prod_{p=1}^{t-1} A_{p} . Y_{t}+\sum_{p \geq t+1} d_{p} . Y_{p}
$$

This implies that $f_{1}\left(Y_{m}\right)=d_{1} \prod_{p=1}^{m-1} A_{p} Y_{m}$. For this vector to be non zero, we must have $d_{1} \prod_{p=1}^{m-1} \neq 0$ This prove that

$$
f\left(Y_{t}\right)=d_{1} \prod_{p=1}^{t-1} A_{p} . Y_{t}+\sum_{p \geq t+1} d_{p} . Y_{p}
$$

start we a non zero componant on $Y_{t}$, and then the images of the vectors $Y_{t}$ by $f$ form a bases.

Conversely, let $f_{0}$ be an adapted change of bases of filiform Lie algebras. Then the map $f=f_{0}+f_{1}$, with $f_{1}$ given by:

$$
\left\{\begin{array}{l}
f_{1}\left(Y_{1}\right)=d_{1} Y_{1}+d_{2} Y_{2}+\ldots d_{m} Y_{m} \\
f_{1}\left(Y_{i}\right)=\left[f_{0}\left(X_{0}\right), f_{1}\left(Y_{i-1}\right)\right] \quad 2 \leq i \leq m
\end{array}\right.
$$


and the condition:

$$
d_{1} \prod_{j=1}^{m-1} A_{j} \neq 0
$$

is an adapted change of basis.

\subsection{Classification in low dimensions}

We have a description of the products of the filiform Lie superalgebras on $\mathcal{F}_{1, m}$. There exists two types:

(1)

$$
\left\{\begin{array}{l}
{\left[X_{0}, Y_{i}\right]=Y_{i+1}, \quad 1 \leq i \leq m-1} \\
{\left[Y_{i}, Y_{j}\right]=(-1)^{\frac{i-j}{2}} a_{\frac{i+j}{2}} X_{1}, \text { if } i+j \text { even and } 2 \leq i+j \leq m+1}
\end{array}\right.
$$

the other products vanish.

$(2)$

$$
\left\{\begin{array}{l}
{\left[X_{0}, Y_{i}\right]=Y_{i+1}, \quad 1 \leq i \leq m-1} \\
{\left[X_{1}, Y_{r}\right]=\sum_{s=2}^{m} r_{s} Y_{s+r-1} \text { with } s+r-1 \leq m}
\end{array}\right.
$$

the other products vanish.

Using adapted changes of bases, like it was done for Lie algebras in [4], we can eliminate the parameters $a_{\frac{i+j}{2}}$.

Theorem 3.1. Every filiform Lie superalgebra of $\mathcal{F}_{1, m}$ which is not a Lie algebra is isomorphic to one of the following filiform Lie superalgebras:

$$
\begin{aligned}
& {\left[X_{0}, Y_{i}\right]=Y_{i+1}, \quad 1 \leq i \leq m-1} \\
& {\left[Y_{i}, Y_{2 k-i}\right]=(-1)^{k-i} X_{1} \quad 1 \leq i \leq k}
\end{aligned}
$$

with $1 \leq k \leq z+1$ if $m=2 z+1$ and $1 \leq k \leq z$ if $m=2 z$.

Proof. We can assume that every non trivial filiform Lie superalgebra with $1 \leq p \leq k-1$ is isomorphic to:

$$
\begin{aligned}
& {\left[X_{0}, Y_{i}\right]=Y_{i+1}, \quad 1 \leq i \leq m-1} \\
& {\left[Y_{i}, Y_{2 k-i}\right]=(-1)^{k-i} X_{1} \quad 1 \leq i \leq k} \\
& {\left[Y_{i}, Y_{j}\right]=(-1)^{\frac{i-j}{2}} a_{\frac{i+j}{2}} X_{1}, \text { if } i+j \text { even and } 2 \leq i+j \leq 2(k-p)}
\end{aligned}
$$


for $1 \leq p \leq k-1$. The other products vanish.

Using this change of adapted bases:

$$
\left\{\begin{array}{l}
X_{0}^{1}=X_{0} \\
X_{1}^{1}=X_{1} \\
Y_{1}^{1}=Y_{1}-(-1)^{p} \frac{a_{k-1}}{2} Y_{2 p+1} \\
\cdots \\
Y_{k-p}^{1}=Y_{k-p}-(-1)^{p} \frac{a_{k-1}}{2} Y_{k+p} \\
Y_{k-p+s}^{1}=Y_{k-p+s}-(-1)^{p} \frac{a_{k-1}}{2} Y_{k+p+s}
\end{array}\right.
$$

we can reduce to

$$
\left\{\begin{array}{l}
{\left[X_{0}, Y_{i}\right]=Y_{i+1}, \quad 1 \leq i \leq m-1} \\
{\left[Y_{i}, Y_{2 k-i}\right]=(-1)^{k-i} X_{1} \quad 1 \leq i \leq k} \\
{\left[Y_{i}, Y_{j}\right]=(-1)^{\frac{i-j}{2}} a_{\frac{i+j}{2}} X_{1}, \text { if } i+j \text { even and } 2 \leq i+j \leq 2(k-p-1)}
\end{array}\right.
$$

The other products vanish.

By induction on $p$, we have a complete classification.

By using adapted changes of bases, we established the following classifications:

$\mathcal{F}_{1,2}:$

(1) $\left\{\left[X_{0}, Y_{1}\right]=Y_{2}\right.$

(2) $\left\{\begin{array}{l}{\left[X_{0}, Y_{1}\right]=Y_{2}} \\ {\left[X_{1}, Y_{1}\right]=Y_{2}}\end{array}\right.$

(3) $\left\{\begin{array}{l}{\left[X_{0}, Y_{1}\right]=Y_{2}} \\ {\left[Y_{1}, Y_{1}\right]=X_{1}}\end{array}\right.$

$\mathcal{F}_{1,3}$ :

(1) $\left\{\left[X_{0}, Y_{1}\right]=Y_{2}, \quad\left[X_{0}, Y_{2}\right]=Y_{3}\right.$

(2) $\left\{\begin{array}{l}{\left[X_{0}, Y_{1}\right]=Y_{2}, \quad\left[X_{0}, Y_{2}\right]=Y_{3}} \\ {\left[Y_{1}, Y_{1}\right]=X_{1}}\end{array}\right.$ 

(3) $\begin{cases}{\left[X_{0}, Y_{1}\right]=Y_{2},} & {\left[X_{0}, Y_{2}\right]=Y_{3}} \\ {\left[Y_{2}, Y_{2}\right]=X_{1},} & {\left[Y_{1}, Y_{3}\right]=-X_{1}}\end{cases}$
(4) $\begin{cases}{\left[X_{0}, Y_{1}\right]=Y_{2},} & {\left[X_{0}, Y_{2}\right]=Y_{3}} \\ {\left[X_{1}, Y_{1}\right]=Y_{2},} & {\left[X_{1}, Y_{2}\right]=Y_{3}}\end{cases}$
(5) $\left\{\begin{array}{l}{\left[X_{0}, Y_{1}\right]=Y_{2}, \quad\left[X_{0}, Y_{2}\right]=Y_{3}} \\ {\left[X_{1}, Y_{1}\right]=Y_{3}}\end{array}\right.$
(6) $\left\{\begin{array}{l}{\left[X_{0}, Y_{1}\right]=Y_{2}, \quad\left[X_{0}, Y_{2}\right]=Y_{3}} \\ {\left[X_{1}, Y_{1}\right]=Y_{2}+Y_{3}, \quad\left[X_{1}, Y_{2}\right]=Y_{3}}\end{array}\right.$

$\mathcal{F}_{1,4}:$

(1) $\left\{\left[X_{0}, Y_{1}\right]=Y_{2}, \quad\left[X_{0}, Y_{2}\right]=Y_{3}, \quad\left[X_{0}, Y_{3}\right]=Y_{4}\right.$

(2) $\left\{\begin{array}{l}{\left[X_{0}, Y_{1}\right]=Y_{2}, \quad\left[X_{0}, Y_{2}\right]=Y_{3}, \quad\left[X_{0}, Y_{3}\right]=Y_{4}} \\ {\left[Y_{1}, Y_{1}\right]=X_{1}}\end{array}\right.$

(3) $\left\{\begin{array}{lll}{\left[X_{0}, Y_{1}\right]=Y_{2},} & {\left[X_{0}, Y_{2}\right]=Y_{3}, \quad\left[X_{0}, Y_{3}\right]=Y_{4}} \\ {\left[Y_{2}, Y_{2}\right]=X_{1},} & {\left[Y_{1}, Y_{3}\right]=-X_{1}}\end{array}\right.$

(4) $\left\{\begin{array}{lll}{\left[X_{0}, Y_{1}\right]=Y_{2},} & {\left[X_{0}, Y_{2}\right]=Y_{3},} & {\left[X_{0}, Y_{3}\right]=Y_{4}} \\ {\left[X_{1}, Y_{1}\right]=Y_{2},} & {\left[X_{1}, Y_{2}\right]=Y_{3},} & {\left[X_{1}, Y_{3}\right]=Y_{4}}\end{array}\right.$

(5) $\left\{\begin{array}{lll}{\left[X_{0}, Y_{1}\right]=Y_{2},} & {\left[X_{0}, Y_{2}\right]=Y_{3},} & {\left[X_{0}, Y_{3}\right]=Y_{4}} \\ {\left[X_{1}, Y_{1}\right]=Y_{3},} & {\left[X_{1}, Y_{2}\right]=Y_{4}} & \end{array}\right.$

(6) $\left\{\begin{array}{l}{\left[X_{0}, Y_{1}\right]=Y_{2}, \quad\left[X_{0}, Y_{2}\right]=Y_{3}, \quad\left[X_{0}, Y_{3}\right]=Y_{4}} \\ {\left[X_{1}, Y_{1}\right]=Y_{4}}\end{array}\right.$

(7) $\left\{\begin{array}{l}{\left[X_{0}, Y_{1}\right]=Y_{2}, \quad\left[X_{0}, Y_{2}\right]=Y_{3}, \quad\left[X_{0}, Y_{3}\right]=Y_{4}} \\ {\left[X_{1}, Y_{1}\right]=Y_{2}+Y_{3}, \quad\left[X_{1}, Y_{2}\right]=Y_{3}+Y_{4}, \quad\left[X_{1}, Y_{3}\right]=Y_{4}}\end{array}\right.$

(8) $\left\{\begin{array}{l}{\left[X_{0}, Y_{1}\right]=Y_{2}, \quad\left[X_{0}, Y_{2}\right]=Y_{3}, \quad\left[X_{0}, Y_{3}\right]=Y_{4}} \\ {\left[X_{1}, Y_{1}\right]=Y_{2}+Y_{4}, \quad\left[X_{1}, Y_{2}\right]=Y_{3}, \quad\left[X_{1}, Y_{3}\right]=Y_{4}}\end{array}\right.$

(9) $\left\{\begin{array}{l}{\left[X_{0}, Y_{1}\right]=Y_{2}, \quad\left[X_{0}, Y_{2}\right]=Y_{3}, \quad\left[X_{0}, Y_{3}\right]=Y_{4}} \\ {\left[X_{1}, Y_{1}\right]=Y_{2}+Y_{3}+2 Y_{4}, \quad\left[X_{1}, Y_{2}\right]=Y_{3}+Y_{4}, \quad\left[X_{1}, Y_{3}\right]=Y_{4}}\end{array}\right.$

$\mathcal{F}_{2,2}$ :

(1) $\left\{\left[X_{0}, X_{1}\right]=X_{2}, \quad\left[X_{0}, Y_{1}\right]=Y_{2}\right.$ 

(2) $\left\{\begin{array}{l}{\left[X_{0}, X_{1}\right]=X_{2}, \quad\left[X_{0}, Y_{1}\right]=Y_{2}} \\ {\left[Y_{1}, Y_{1}\right]=X_{1}, \quad\left[Y_{1}, Y_{2}\right]=\frac{1}{2} X_{2}}\end{array}\right.$
(3) $\left\{\begin{array}{l}{\left[X_{0}, X_{1}\right]=X_{2}, \quad\left[X_{0}, Y_{1}\right]=Y_{2}} \\ {\left[Y_{1}, Y_{1}\right]=X_{2}}\end{array}\right.$
(4) $\left\{\begin{array}{l}{\left[X_{0}, X_{1}\right]=X_{2}, \quad\left[X_{0}, Y_{1}\right]=Y_{2}} \\ {\left[X_{1}, Y_{1}\right]=Y_{2}}\end{array}\right.$
(5) $\begin{cases}{\left[X_{0}, X_{1}\right]=X_{2},} & {\left[X_{0}, Y_{1}\right]=Y_{2}} \\ {\left[X_{1}, Y_{1}\right]=Y_{2},} & {\left[Y_{1}, Y_{1}\right]=X_{2}}\end{cases}$

$\mathcal{F}_{2,3}$ :

(1) $\left\{\left[X_{0}, X_{1}\right]=X_{2}, \quad\left[X_{0}, Y_{1}\right]=Y_{2}, \quad\left[X_{0}, Y_{2}\right]=Y_{3}\right.$

(2) $\left\{\begin{array}{l}{\left[X_{0}, X_{1}\right]=X_{2}, \quad\left[X_{0}, Y_{1}\right]=Y_{2}, \quad\left[X_{0}, Y_{2}\right]=Y_{3}} \\ {\left[X_{1}, Y_{1}\right]=Y_{2}, \quad\left[X_{1}, Y_{2}\right]=Y_{3}}\end{array}\right.$

(3) $\left\{\begin{array}{l}{\left[X_{0}, X_{1}\right]=X_{2}, \quad\left[X_{0}, Y_{1}\right]=Y_{2}, \quad\left[X_{0}, Y_{2}\right]=Y_{3}} \\ {\left[X_{1}, Y_{1}\right]=Y_{3}}\end{array}\right.$

(4) $\left\{\begin{array}{l}{\left[X_{0}, X_{1}\right]=X_{2}, \quad\left[X_{0}, Y_{1}\right]=Y_{2}, \quad\left[X_{0}, Y_{2}\right]=Y_{3}} \\ {\left[X_{1}, Y_{2}\right]=-Y_{3}, \quad\left[X_{2}, Y_{1}\right]=Y_{3}}\end{array}\right.$

(5) $\left\{\begin{array}{l}{\left[X_{0}, X_{1}\right]=X_{2}, \quad\left[X_{0}, Y_{1}\right]=Y_{2}, \quad\left[X_{0}, Y_{2}\right]=Y_{3}} \\ {\left[X_{1}, Y_{1}\right]=Y_{2}+Y_{3}, \quad\left[X_{1}, Y_{2}\right]=Y_{3}}\end{array}\right.$

(6) $\left\{\begin{array}{l}{\left[X_{0}, X_{1}\right]=X_{2}, \quad\left[X_{0}, Y_{1}\right]=Y_{2}, \quad\left[X_{0}, Y_{2}\right]=Y_{3}} \\ {\left[X_{1}, Y_{1}\right]=Y_{2}, \quad\left[X_{2}, Y_{1}\right]=Y_{3}}\end{array}\right.$

(7) $\left\{\begin{array}{lll}{\left[X_{0}, X_{1}\right]=X_{2},} & {\left[X_{0}, Y_{1}\right]=Y_{2},} & {\left[X_{0}, Y_{2}\right]=Y_{3}} \\ {\left[X_{1}, Y_{1}\right]=2 Y_{2},} & {\left[X_{1}, Y_{2}\right]=Y_{3},} & {\left[X_{2}, Y_{1}\right]=Y_{3}}\end{array}\right.$

(8) $\left\{\begin{array}{l}{\left[X_{0}, X_{1}\right]=X_{2}, \quad\left[X_{0}, Y_{1}\right]=Y_{2}, \quad\left[X_{0}, Y_{2}\right]=Y_{3}} \\ {\left[Y_{1}, Y_{1}\right]=X_{2}}\end{array}\right.$

(9) $\left\{\begin{array}{l}{\left[X_{0}, X_{1}\right]=X_{2}, \quad\left[X_{0}, Y_{1}\right]=Y_{2}, \quad\left[X_{0}, Y_{2}\right]=Y_{3}} \\ {\left[X_{1}, Y_{1}\right]=Y_{2}, \quad\left[X_{1}, Y_{2}\right]=Y_{3}} \\ {\left[Y_{1}, Y_{1}\right]=X_{2}}\end{array}\right.$ 
(10) $\left\{\begin{array}{l}{\left[X_{0}, X_{1}\right]=X_{2}, \quad\left[X_{0}, Y_{1}\right]=Y_{2}, \quad\left[X_{0}, Y_{2}\right]=Y_{3}} \\ {\left[X_{1}, Y_{1}\right]=Y_{3}} \\ {\left[Y_{1}, Y_{1}\right]=X_{2}}\end{array}\right.$

(11) $\left\{\begin{array}{l}{\left[X_{0}, X_{1}\right]=X_{2}, \quad\left[X_{0}, Y_{1}\right]=Y_{2}, \quad\left[X_{0}, Y_{2}\right]=Y_{3}} \\ {\left[X_{1}, Y_{1}\right]=Y_{2}+Y_{3}, \quad\left[X_{1}, Y_{2}\right]=Y_{3}} \\ {\left[Y_{1}, Y_{1}\right]=X_{2}}\end{array}\right.$

(12) $\begin{cases}{\left[X_{0}, X_{1}\right]=X_{2},} & {\left[X_{0}, Y_{1}\right]=Y_{2}, \quad\left[X_{0}, Y_{2}\right]=Y_{3}} \\ {\left[Y_{1}, Y_{3}\right]=-X_{2},} & {\left[Y_{2}, Y_{2}\right]=X_{2}}\end{cases}$

(13) $\left\{\begin{array}{l}{\left[X_{0}, X_{1}\right]=X_{2}, \quad\left[X_{0}, Y_{1}\right]=Y_{2}, \quad\left[X_{0}, Y_{2}\right]=Y_{3}} \\ {\left[X_{1}, Y_{1}\right]=Y_{2}, \quad\left[X_{1}, Y_{2}\right]=Y_{3}} \\ {\left[Y_{1}, Y_{3}\right]=-X_{2}, \quad\left[Y_{2}, Y_{2}\right]=X_{2}}\end{array}\right.$

(14) $\left\{\begin{array}{l}{\left[X_{0}, X_{1}\right]=X_{2}, \quad\left[X_{0}, Y_{1}\right]=Y_{2}, \quad\left[X_{0}, Y_{2}\right]=Y_{3}} \\ {\left[Y_{1}, Y_{1}\right]=X_{1}, \quad\left[Y_{1}, Y_{2}\right]=\frac{1}{2} X_{2}}\end{array}\right.$

(15) $\left\{\begin{array}{l}{\left[X_{0}, X_{1}\right]=X_{2}, \quad\left[X_{0}, Y_{1}\right]=Y_{2}, \quad\left[X_{0}, Y_{2}\right]=Y_{3}} \\ {\left[X_{1}, Y_{2}\right]=-Y_{3}, \quad\left[X_{2}, Y_{1}\right]=Y_{3}} \\ {\left[Y_{1}, Y_{1}\right]=X_{1}, \quad\left[Y_{1}, Y_{2}\right]=\frac{1}{2} X_{2}}\end{array}\right.$

(16) $\left\{\begin{array}{lll}{\left[X_{0}, X_{1}\right]=X_{2},} & {\left[X_{0}, Y_{1}\right]=Y_{2},} & {\left[X_{0}, Y_{2}\right]=Y_{3}} \\ {\left[Y_{1}, Y_{1}\right]=X_{1},} & {\left[Y_{1}, Y_{2}\right]=\frac{1}{2} X_{2},} & {\left[Y_{1}, Y_{3}\right]=-X_{2}, \quad\left[Y_{2}, Y_{2}\right]=X_{2}}\end{array}\right.$

$\mathcal{F}_{3,2}$ :

(1) $\left\{\left[X_{0}, X_{1}\right]=X_{2}, \quad\left[X_{0}, X_{2}\right]=X_{3}, \quad\left[X_{0}, Y_{1}\right]=Y_{2}\right.$

(2) $\left\{\begin{array}{lcc}{\left[X_{0}, X_{1}\right]=X_{2},} & {\left[X_{0}, X_{2}\right]=X_{3},} & {\left[X_{0}, Y_{1}\right]=Y_{2}} \\ {\left[Y_{1}, Y_{1}\right]=X_{1},} & {\left[Y_{1}, Y_{2}\right]=\frac{1}{2} X_{2},} & {\left[Y_{2}, Y_{2}\right]=\frac{1}{2} X_{3}}\end{array}\right.$

(3) $\left\{\begin{array}{l}{\left[X_{0}, X_{1}\right]=X_{2}, \quad\left[X_{0}, X_{2}\right]=X_{3}, \quad\left[X_{0}, Y_{1}\right]=Y_{2}} \\ {\left[Y_{1}, Y_{1}\right]=X_{2}, \quad\left[Y_{1}, Y_{2}\right]=\frac{1}{2} X_{3}}\end{array}\right.$

(4) $\left\{\begin{array}{l}{\left[X_{0}, X_{1}\right]=X_{2}, \quad\left[X_{0}, X_{2}\right]=X_{3}, \quad\left[X_{0}, Y_{1}\right]=Y_{2}} \\ {\left[Y_{1}, Y_{1}\right]=X_{3}}\end{array}\right.$

(5) $\left\{\begin{array}{l}{\left[X_{0}, X_{1}\right]=X_{2}, \quad\left[X_{0}, X_{2}\right]=X_{3}, \quad\left[X_{0}, Y_{1}\right]=Y_{2}} \\ {\left[X_{1}, Y_{1}\right]=Y_{2}}\end{array}\right.$ 
(6) $\left\{\begin{array}{l}{\left[X_{0}, X_{1}\right]=X_{2}, \quad\left[X_{0}, X_{2}\right]=X_{3}, \quad\left[X_{0}, Y_{1}\right]=Y_{2}} \\ {\left[X_{1}, Y_{1}\right]=Y_{2}, \quad\left[Y_{1}, Y_{1}\right]=X_{3}}\end{array}\right.$ $\mathcal{F}_{4,2}$ :

(1) $\left\{\left[X_{0}, X_{1}\right]=X_{2}, \quad\left[X_{0}, X_{2}\right]=X_{3}, \quad\left[X_{0}, X_{3}\right]=X_{4}, \quad\left[X_{0}, Y_{1}\right]=Y_{2}\right.$

(2) $\left\{\begin{array}{lll}{\left[X_{0}, X_{1}\right]=X_{2},} & {\left[X_{0}, X_{2}\right]=X_{3},} & {\left[X_{0}, X_{3}\right]=X_{4}, \quad\left[X_{0}, Y_{1}\right]=Y_{2}} \\ {\left[Y_{1}, Y_{1}\right]=X_{2},} & {\left[Y_{1}, Y_{2}\right]=\frac{1}{2} X_{3},} & {\left[Y_{2}, Y_{2}\right]=\frac{1}{2} X_{4}}\end{array}\right.$

(3) $\left\{\begin{array}{l}{\left[X_{0}, X_{1}\right]=X_{2}, \quad\left[X_{0}, X_{2}\right]=X_{3}, \quad\left[X_{0}, X_{3}\right]=X_{4}, \quad\left[X_{0}, Y_{1}\right]=Y_{2}} \\ {\left[Y_{1}, Y_{1}\right]=X_{3}, \quad\left[Y_{1}, Y_{2}\right]=\frac{1}{2} X_{4}}\end{array}\right.$

(4) $\left\{\begin{array}{l}{\left[X_{0}, X_{1}\right]=X_{2}, \quad\left[X_{0}, X_{2}\right]=X_{3}, \quad\left[X_{0}, X_{3}\right]=X_{4}, \quad\left[X_{0}, Y_{1}\right]=Y_{2}} \\ {\left[Y_{1}, Y_{1}\right]=X_{4}}\end{array}\right.$

(5) $\left\{\begin{array}{l}{\left[X_{0}, X_{1}\right]=X_{2}, \quad\left[X_{0}, X_{2}\right]=X_{3}, \quad\left[X_{0}, X_{3}\right]=X_{4}, \quad\left[X_{0}, Y_{1}\right]=Y_{2}} \\ {\left[X_{1}, Y_{1}\right]=Y_{2}}\end{array}\right.$

(6) $\left\{\begin{array}{l}{\left[X_{0}, X_{1}\right]=X_{2}, \quad\left[X_{0}, X_{2}\right]=X_{3}, \quad\left[X_{0}, X_{3}\right]=X_{4}, \quad\left[X_{0}, Y_{1}\right]=Y_{2}} \\ {\left[X_{1}, Y_{1}\right]=Y_{2}, \quad\left[Y_{1}, Y_{1}\right]=X_{4}}\end{array}\right.$

(7) $\left\{\begin{array}{l}{\left[X_{0}, X_{1}\right]=X_{2}, \quad\left[X_{0}, X_{2}\right]=X_{3}, \quad\left[X_{0}, X_{3}\right]=X_{4}, \quad\left[X_{0}, Y_{1}\right]=Y_{2}} \\ {\left[X_{1}, X_{2}\right]=X_{4}}\end{array}\right.$

(8) $\left\{\begin{array}{lll}{\left[X_{0}, X_{1}\right]=X_{2},} & {\left[X_{0}, X_{2}\right]=X_{3},} & {\left[X_{0}, X_{3}\right]=X_{4}, \quad\left[X_{0}, Y_{1}\right]=Y_{2}} \\ {\left[X_{1}, X_{2}\right]=X_{4},} & {\left[Y_{1}, Y_{1}\right]=X_{3},} & {\left[Y_{1}, Y_{2}\right]=\frac{1}{2} X_{4}}\end{array}\right.$

(9) $\left\{\begin{array}{lll}{\left[X_{0}, X_{1}\right]=X_{2},} & {\left[X_{0}, X_{2}\right]=X_{3}, \quad\left[X_{0}, X_{3}\right]=X_{4}, \quad\left[X_{0}, Y_{1}\right]=Y_{2}} \\ {\left[X_{1}, X_{2}\right]=X_{4},} & {\left[Y_{1}, Y_{1}\right]=X_{4}}\end{array}\right.$

(10) $\begin{cases}{\left[X_{0}, X_{1}\right]=X_{2},} & {\left[X_{0}, X_{2}\right]=X_{3}, \quad\left[X_{0}, X_{3}\right]=X_{4}, \quad\left[X_{0}, Y_{1}\right]=Y_{2}} \\ {\left[X_{1}, X_{2}\right]=X_{4},} & {\left[X_{1}, Y_{1}\right]=Y_{2}}\end{cases}$

(11) $\left\{\begin{array}{lll}{\left[X_{0}, X_{1}\right]=X_{2},} & {\left[X_{0}, X_{2}\right]=X_{3}, \quad\left[X_{0}, X_{3}\right]=X_{4}, \quad\left[X_{0}, Y_{1}\right]=Y_{2}} \\ {\left[X_{1}, X_{2}\right]=X_{4},} & {\left[X_{1}, Y_{1}\right]=Y_{2},} & {\left[Y_{1}, Y_{1}\right]=X_{4}}\end{array}\right.$

$\mathcal{F}_{5,2}$ :

(1) $\left\{\begin{array}{l}{\left[X_{0}, X_{1}\right]=X_{2}, \quad\left[X_{0}, X_{2}\right]=X_{3}, \quad\left[X_{0}, X_{3}\right]=X_{4}, \quad\left[X_{0}, X_{4}\right]=X_{5}} \\ {\left[X_{0}, Y_{1}\right]=Y_{2}}\end{array}\right.$ 

(2) $\left\{\begin{array}{l}{\left[X_{0}, X_{1}\right]=X_{2}, \quad\left[X_{0}, X_{2}\right]=X_{3}, \quad\left[X_{0}, X_{3}\right]=X_{4}, \quad\left[X_{0}, X_{4}\right]=X_{5}} \\ {\left[X_{0}, Y_{1}\right]=Y_{2}} \\ {\left[Y_{1}, Y_{1}\right]=X_{3}, \quad\left[Y_{1}, Y_{2}\right]=\frac{1}{2} X_{4}, \quad\left[Y_{2}, Y_{2}\right]=\frac{1}{2} X_{5}}\end{array}\right.$
(3) $\left\{\begin{array}{l}{\left[X_{0}, X_{1}\right]=X_{2}, \quad\left[X_{0}, X_{2}\right]=X_{3}, \quad\left[X_{0}, X_{3}\right]=X_{4}, \quad\left[X_{0}, X_{4}\right]=X_{5}} \\ {\left[X_{0}, Y_{1}\right]=Y_{2}} \\ {\left[Y_{1}, Y_{1}\right]=X_{4}, \quad\left[Y_{1}, Y_{2}\right]=\frac{1}{2} X_{5}}\end{array}\right.$
(4) $\left\{\begin{array}{l}{\left[X_{0}, X_{1}\right]=X_{2}, \quad\left[X_{0}, X_{2}\right]=X_{3}, \quad\left[X_{0}, X_{3}\right]=X_{4}, \quad\left[X_{0}, X_{4}\right]=X_{5}} \\ {\left[X_{0}, Y_{1}\right]=Y_{2}} \\ {\left[Y_{1}, Y_{1}\right]=X_{5}}\end{array}\right.$
(5) $\left\{\begin{array}{l}{\left[X_{0}, X_{1}\right]=X_{2}, \quad\left[X_{0}, X_{2}\right]=X_{3}, \quad\left[X_{0}, X_{3}\right]=X_{4}, \quad\left[X_{0}, X_{4}\right]=X_{5}} \\ {\left[X_{0}, Y_{1}\right]=Y_{2}} \\ {\left[X_{1}, Y_{1}\right]=Y_{2}}\end{array}\right.$
(6) $\left\{\begin{array}{l}{\left[X_{0}, X_{1}\right]=X_{2}, \quad\left[X_{0}, X_{2}\right]=X_{3}, \quad\left[X_{0}, X_{3}\right]=X_{4}, \quad\left[X_{0}, X_{4}\right]=X_{5}} \\ {\left[X_{0}, Y_{1}\right]=Y_{2}} \\ {\left[X_{1}, Y_{1}\right]=Y_{2}, \quad\left[Y_{1}, Y_{1}\right]=X_{5}}\end{array}\right.$
(7) $\left\{\begin{array}{l}{\left[X_{0}, X_{1}\right]=X_{2}, \quad\left[X_{0}, X_{2}\right]=X_{3}, \quad\left[X_{0}, X_{3}\right]=X_{4}, \quad\left[X_{0}, X_{4}\right]=X_{5}} \\ {\left[X_{0}, Y_{1}\right]=Y_{2}} \\ {\left[X_{1}, X_{2}\right]=X_{5}}\end{array}\right.$

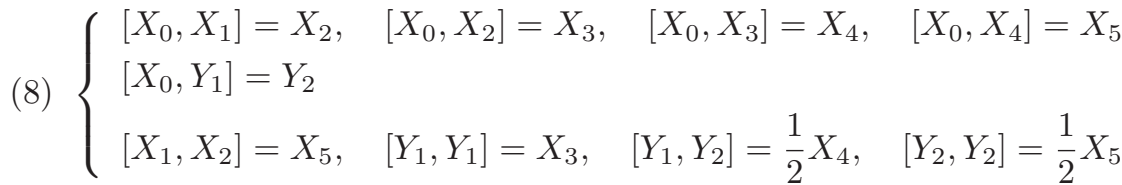
(9) $\left\{\begin{array}{lll}{\left[X_{0}, X_{1}\right]=X_{2},} & {\left[X_{0}, X_{2}\right]=X_{3},} & {\left[X_{0}, X_{3}\right]=X_{4}, \quad\left[X_{0}, X_{4}\right]=X_{5}} \\ {\left[X_{0}, Y_{1}\right]=Y_{2}} & \\ {\left[X_{1}, X_{2}\right]=X_{5},} & {\left[Y_{1}, Y_{1}\right]=X_{4},} & {\left[Y_{1}, Y_{2}\right]=\frac{1}{2} X_{5}}\end{array}\right.$
(10) $\left\{\begin{array}{l}{\left[X_{0}, X_{1}\right]=X_{2}, \quad\left[X_{0}, X_{2}\right]=X_{3}, \quad\left[X_{0}, X_{3}\right]=X_{4}, \quad\left[X_{0}, X_{4}\right]=X_{5}} \\ {\left[X_{0}, Y_{1}\right]=Y_{2}} \\ {\left[X_{1}, X_{2}\right]=X_{5}, \quad\left[Y_{1}, Y_{1}\right]=X_{5}}\end{array}\right.$
(11) $\left\{\begin{array}{l}{\left[X_{0}, X_{1}\right]=X_{2}, \quad\left[X_{0}, X_{2}\right]=X_{3}, \quad\left[X_{0}, X_{3}\right]=X_{4}, \quad\left[X_{0}, X_{4}\right]=X_{5}} \\ {\left[X_{0}, Y_{1}\right]=Y_{2}} \\ {\left[X_{1}, X_{2}\right]=X_{5}, \quad\left[X_{1}, Y_{1}\right]=Y_{2}}\end{array}\right.$ 
(12)

$\left\{\begin{array}{lll}{\left[X_{0}, X_{1}\right]=X_{2},} & {\left[X_{0}, X_{2}\right]=X_{3}, \quad\left[X_{0}, X_{3}\right]=X_{4}, \quad\left[X_{0}, X_{4}\right]=X_{5}} \\ {\left[X_{0}, Y_{1}\right]=Y_{2}} & \\ {\left[X_{1}, X_{2}\right]=X_{5}, \quad\left[X_{1}, Y_{1}\right]=Y_{2},} & {\left[Y_{1}, Y_{1}\right]=X_{5}}\end{array}\right.$

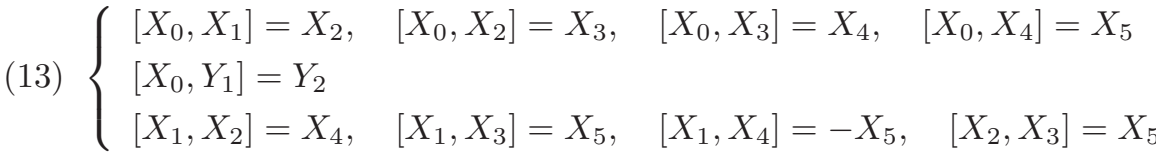

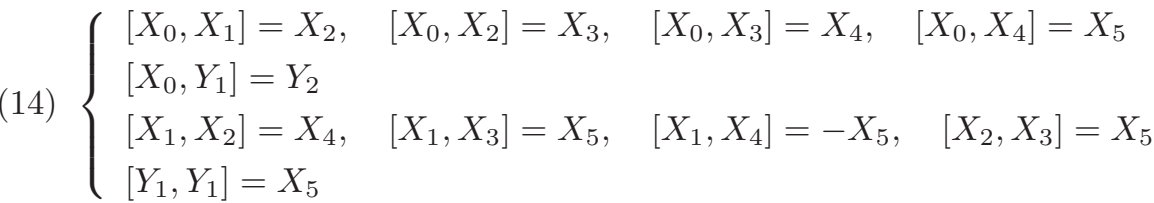

(15) $\left\{\begin{array}{l}{\left[X_{0}, X_{1}\right]=X_{2}, \quad\left[X_{0}, X_{2}\right]=X_{3}, \quad\left[X_{0}, X_{3}\right]=X_{4}, \quad\left[X_{0}, X_{4}\right]=X_{5}} \\ {\left[X_{0}, Y_{1}\right]=Y_{2}} \\ {\left[X_{1}, X_{2}\right]=X_{4}, \quad\left[X_{1}, X_{3}\right]=X_{5}, \quad\left[X_{1}, X_{4}\right]=-X_{5}, \quad\left[X_{2}, X_{3}\right]=X_{5}} \\ {\left[X_{1}, Y_{1}\right]=Y_{2}}\end{array}\right.$

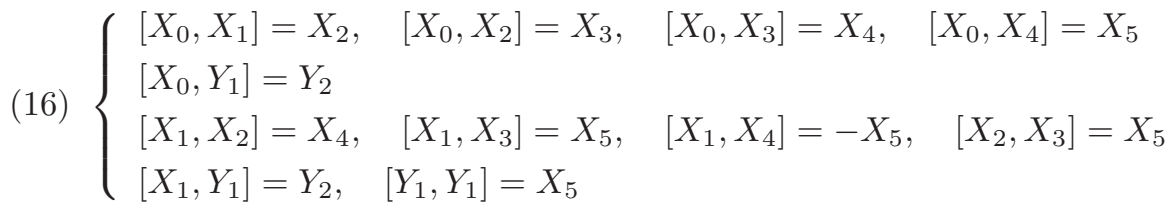

(17) $\left\{\begin{array}{l}{\left[X_{0}, X_{1}\right]=X_{2}, \quad\left[X_{0}, X_{2}\right]=X_{3}, \quad\left[X_{0}, X_{3}\right]=X_{4}, \quad\left[X_{0}, X_{4}\right]=X_{5}} \\ {\left[X_{0}, Y_{1}\right]=Y_{2}} \\ {\left[X_{1}, X_{4}\right]=-X_{5}, \quad\left[X_{2}, X_{3}\right]=X_{5}}\end{array}\right.$

(18) $\left\{\begin{array}{l}{\left[X_{0}, X_{1}\right]=X_{2}, \quad\left[X_{0}, X_{2}\right]=X_{3}, \quad\left[X_{0}, X_{3}\right]=X_{4}, \quad\left[X_{0}, X_{4}\right]=X_{5}} \\ {\left[X_{0}, Y_{1}\right]=Y_{2}} \\ {\left[X_{1}, X_{4}\right]=-X_{5}, \quad\left[X_{2}, X_{3}\right]=X_{5}, \quad\left[Y_{1}, Y_{1}\right]=X_{5}}\end{array}\right.$

(19) $\left\{\begin{array}{l}{\left[X_{0}, X_{1}\right]=X_{2}, \quad\left[X_{0}, X_{2}\right]=X_{3}, \quad\left[X_{0}, X_{3}\right]=X_{4}, \quad\left[X_{0}, X_{4}\right]=X_{5}} \\ {\left[X_{0}, Y_{1}\right]=Y_{2}} \\ {\left[X_{1}, X_{4}\right]=-X_{5}, \quad\left[X_{2}, X_{3}\right]=X_{5}, \quad\left[X_{1}, Y_{1}\right]=Y_{2}}\end{array}\right.$

(20) $\left\{\begin{array}{l}{\left[X_{0}, X_{1}\right]=X_{2}, \quad\left[X_{0}, X_{2}\right]=X_{3}, \quad\left[X_{0}, X_{3}\right]=X_{4}, \quad\left[X_{0}, X_{4}\right]=X_{5}} \\ {\left[X_{0}, Y_{1}\right]=Y_{2}} \\ {\left[X_{1}, X_{4}\right]=-X_{5}, \quad\left[X_{2}, X_{3}\right]=X_{5}, \quad\left[X_{1}, Y_{1}\right]=Y_{2}, \quad\left[Y_{1}, Y_{1}\right]=X_{5}}\end{array}\right.$

(21) $\left\{\begin{array}{l}{\left[X_{0}, X_{1}\right]=X_{2}, \quad\left[X_{0}, X_{2}\right]=X_{3}, \quad\left[X_{0}, X_{3}\right]=X_{4}, \quad\left[X_{0}, X_{4}\right]=X_{5}} \\ {\left[X_{0}, Y_{1}\right]=Y_{2}} \\ {\left[X_{1}, X_{4}\right]=-X_{5}, \quad\left[X_{2}, X_{3}\right]=X_{5}, \quad\left[X_{1}, Y_{1}\right]=-Y_{2}}\end{array}\right.$ 
(22)

$\left\{\begin{array}{l}{\left[X_{0}, X_{1}\right]=X_{2}, \quad\left[X_{0}, X_{2}\right]=X_{3}, \quad\left[X_{0}, X_{3}\right]=X_{4}, \quad\left[X_{0}, X_{4}\right]=X_{5}} \\ {\left[X_{0}, Y_{1}\right]=Y_{2}, \quad\left[X_{1}, X_{4}\right]=-X_{5}} \\ {\left[X_{2}, X_{3}\right]=X_{5}, \quad\left[X_{1}, Y_{1}\right]=-Y_{2}, \quad\left[Y_{1}, Y_{1}\right]=X_{4}, \quad\left[Y_{1}, Y_{2}\right]=\frac{1}{2} X_{5}}\end{array}\right.$

(23) $\left\{\begin{array}{l}{\left[X_{0}, X_{1}\right]=X_{2}, \quad\left[X_{0}, X_{2}\right]=X_{3}, \quad\left[X_{0}, X_{3}\right]=X_{4}, \quad\left[X_{0}, X_{4}\right]=X_{5}} \\ {\left[X_{0}, Y_{1}\right]=Y_{2}}\end{array}\right.$ $\left[X_{1}, X_{4}\right]=-X_{5}, \quad\left[X_{2}, X_{3}\right]=X_{5}, \quad\left[X_{1}, Y_{1}\right]=-Y_{2}, \quad\left[Y_{1}, Y_{1}\right]=X_{5}$

(24) $\left\{\begin{array}{l}{\left[X_{0}, X_{1}\right]=X_{2}, \quad\left[X_{0}, X_{2}\right]=X_{3}, \quad\left[X_{0}, X_{3}\right]=X_{4}, \quad\left[X_{0}, X_{4}\right]=X_{5}} \\ {\left[X_{0}, Y_{1}\right]=Y_{2}} \\ {\left[X_{1}, X_{2}\right]=X_{5}, \quad\left[X_{1}, X_{4}\right]=-X_{5}, \quad\left[X_{2}, X_{3}\right]=X_{5}}\end{array}\right.$

$\left\{\begin{array}{l}{\left[X_{0}, X_{1}\right]=X_{2}, \quad\left[X_{0}, X_{2}\right]=X_{3}, \quad\left[X_{0}, X_{3}\right]=X_{4}, \quad\left[X_{0}, X_{4}\right]=X_{5}} \\ {\left[X_{0}, Y_{1}\right]=Y_{2}} \\ {\left[X_{1}, X_{2}\right]=X_{5}, \quad\left[X_{1}, X_{4}\right]=-X_{5}, \quad\left[X_{2}, X_{3}\right]=X_{5}} \\ {\left[Y_{1}, Y_{1}\right]=X_{5}}\end{array}\right.$

(26) $\left\{\begin{array}{l}\left.\left[X_{0}, X_{1}\right]=X_{2}, \quad\left[X_{0}, X_{2}\right]=X_{3}, \quad\left[X_{0}, X_{3}\right]=X_{4}, \quad\left[X_{0}, X_{4}\right]=X_{5}, Y_{1}\right]=Y_{2} \\ {\left[X_{1}, X_{2}\right]=X_{5}, \quad\left[X_{1}, X_{4}\right]=-X_{5}, \quad\left[X_{2}, X_{3}\right]=X_{5}} \\ {\left[X_{1}, Y_{1}\right]=Y_{2}}\end{array}\right.$

$\left\{\begin{array}{l}{\left[X_{0}, X_{1}\right]=X_{2}, \quad\left[X_{0}, X_{2}\right]=X_{3}, \quad\left[X_{0}, X_{3}\right]=X_{4}, \quad\left[X_{0}, X_{4}\right]=X_{5}} \\ {\left[X_{0}, Y_{1}\right]=Y_{2}} \\ {\left[X_{1}, X_{2}\right]=X_{5}, \quad\left[X_{1}, X_{4}\right]=-X_{5}, \quad\left[X_{2}, X_{3}\right]=X_{5}} \\ {\left[X_{1}, Y_{1}\right]=Y_{2}, \quad\left[Y_{1}, Y_{1}\right]=X_{5}}\end{array}\right.$

(28) $\left\{\begin{array}{l}{\left[X_{0}, X_{1}\right]=X_{2}, \quad\left[X_{0}, X_{2}\right]=X_{3}, \quad\left[X_{0}, X_{3}\right]=X_{4}, \quad\left[X_{0}, X_{4}\right]=X_{5}} \\ {\left[X_{0}, Y_{1}\right]=Y_{2}} \\ {\left[X_{1}, X_{2}\right]=X_{5}, \quad\left[X_{1}, X_{4}\right]=-X_{5}, \quad\left[X_{2}, X_{3}\right]=X_{5}} \\ {\left[X_{1}, Y_{1}\right]=-Y_{2}}\end{array}\right.$

(29) $\left\{\begin{array}{lll}{\left[X_{0}, X_{1}\right]=X_{2},} & {\left[X_{0}, X_{2}\right]=X_{3}, \quad\left[X_{0}, X_{3}\right]=X_{4}, \quad\left[X_{0}, X_{4}\right]=X_{5}} \\ {\left[X_{0}, Y_{1}\right]=Y_{2}} & \\ {\left[X_{1}, X_{2}\right]=X_{5},} & {\left[X_{1}, X_{4}\right]=-X_{5}, \quad\left[X_{2}, X_{3}\right]=X_{5}} \\ {\left[X_{1}, Y_{1}\right]=-Y_{2},} & {\left[Y_{1}, Y_{1}\right]=X_{4}, \quad\left[Y_{1}, Y_{2}\right]=\frac{1}{2} X_{5}}\end{array}\right.$ 
(30)

$\left\{\begin{array}{l}{\left[X_{0}, X_{1}\right]=X_{2}, \quad\left[X_{0}, X_{2}\right]=X_{3}, \quad\left[X_{0}, X_{3}\right]=X_{4}, \quad\left[X_{0}, X_{4}\right]=X_{5}} \\ {\left[X_{0}, Y_{1}\right]=Y_{2}} \\ {\left[X_{1}, X_{2}\right]=X_{5}, \quad\left[X_{1}, X_{4}\right]=-X_{5}, \quad\left[X_{2}, X_{3}\right]=X_{5}} \\ {\left[X_{1}, Y_{1}\right]=-Y_{2}, \quad\left[Y_{1}, Y_{1}\right]=X_{5}}\end{array}\right.$

(31) $\left\{\begin{array}{l}{\left[X_{0}, X_{1}\right]=X_{2}, \quad\left[X_{0}, X_{2}\right]=X_{3}, \quad\left[X_{0}, X_{3}\right]=X_{4}, \quad\left[X_{0}, X_{4}\right]=X_{5}} \\ {\left[X_{0}, Y_{1}\right]=Y_{2}}\end{array}\right.$

$\left[X_{1}, X_{2}\right]=X_{4}+X_{5}, \quad\left[X_{1}, X_{3}\right]=X_{5}, \quad\left[X_{1}, Y_{1}\right]=-2 Y_{2}$

(32) $\left\{\begin{array}{l}{\left[X_{0}, X_{1}\right]=X_{2}, \quad\left[X_{0}, X_{2}\right]=X_{3}, \quad\left[X_{0}, X_{3}\right]=X_{4}, \quad\left[X_{0}, X_{4}\right]=X_{5}} \\ {\left[X_{0}, Y_{1}\right]=Y_{2}} \\ {\left[X_{1}, X_{2}\right]=X_{4}+X_{5},\left[X_{1}, X_{3}\right]=X_{5},\left[X_{1}, Y_{1}\right]=-2 Y_{2},\left[Y_{1}, Y_{1}\right]=X_{5}}\end{array}\right.$

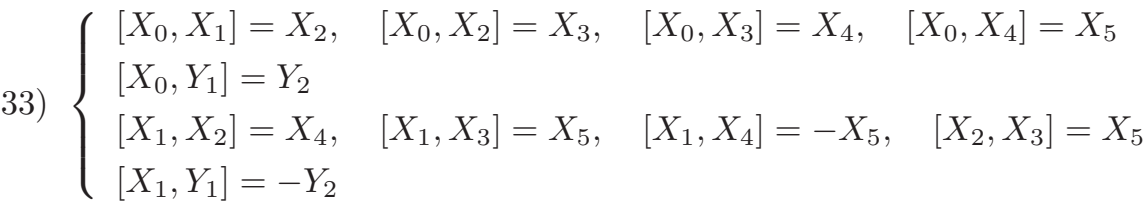

$34)\left\{\begin{array}{llll}{\left[X_{0}, X_{1}\right]=X_{2},} & {\left[X_{0}, X_{2}\right]=X_{3},} & {\left[X_{0}, X_{3}\right]=X_{4},} & {\left[X_{0}, X_{4}\right]=X_{5}} \\ {\left[X_{0}, Y_{1}\right]=Y_{2}} & & \\ {\left[X_{1}, X_{2}\right]=X_{4},} & {\left[X_{1}, X_{3}\right]=X_{5},} & {\left[X_{1}, X_{4}\right]=-X_{5},} & {\left[X_{2}, X_{3}\right]=X_{5}} \\ {\left[X_{1}, Y_{1}\right]=-Y_{2},} & {\left[Y_{1}, Y_{1}\right]=X_{4},} & {\left[Y_{1}, Y_{2}\right]=\frac{1}{2} X_{5}}\end{array}\right.$

$(35)\left\{\begin{array}{lll}{\left[X_{0}, X_{1}\right]=X_{2},} & {\left[X_{0}, X_{2}\right]=X_{3}, \quad\left[X_{0}, X_{3}\right]=X_{4}, \quad\left[X_{0}, X_{4}\right]=X_{5}} \\ {\left[X_{0}, Y_{1}\right]=Y_{2}} & \\ {\left[X_{1}, X_{2}\right]=X_{4},} & {\left[X_{1}, X_{3}\right]=X_{5}, \quad\left[X_{1}, X_{4}\right]=-X_{5}, \quad\left[X_{2}, X_{3}\right]=X_{5}} \\ {\left[X_{1}, Y_{1}\right]=-Y_{2},} & {\left[Y_{1}, Y_{1}\right]=X_{5}} & \end{array}\right.$

\section{References}

[1] J.M. Ancochea-Bermúdez, M. Goze, On the varieties of nilpotent Lie algebras of dimension 7 and 8, J. Pure Appl. Algebra 77 (1992) 131-140

[2] D. B. Fuks Cohomology of Infinite-Dimensional Lie Algebras, Plenum publishing Copr. ISBN 0-306-10990-5

[3] M. Gilg Super-algèbres de Lie nilpotentes Thèse, Université de HauteAlsace, 2000

[4] J.R. Gómez, A. Jimenéz-Merchán, Y. Khakimdjanov Low-dimensional filiform Lie algebras J. of pure and app. Algebras 130 (1998) 133-158 
[5] M. Goze Perturbations des super-algèbres de Lie J. Geom. Phys. vol6, n. 4,1889

[6] M. Scheunert The Theory of Lie Superalgebras Lecture Notes in Math. $716(1979)$

[7] V. G. Kac A Sketch of Lie Superalgebra Theory Commun. math. phys. 53, 31-64 (1977)

[8] V. G. Kac Lie Superalgebras Adv. Math. 26(8), 8-96 (1977)

[9] M. Vergne Cohomologie des algèbres de Lie nilpotentes. Application à l'etude de la variété des algèbres de Lie nilpotentes Bull. Soc. Math. France, 98, 1970 p.81 à 116

[10] G. Vrănceanu Clasificarea grupurilor lui Lie de rang zero Studii şi cercatări mathematice, 1, 1950, 269-308

Université de Haute-Alsace

Laboratoire de Mathématiques

4 rue des Frères Lumière

68093 Mulhouse Cedex, France

E-mail. M.Gilg@univ-mulhouse.fr

Recibido: 26 de Junio de 2000

Revisado: 8 de Enero de 2001 